\title{
MÁ ROTAÇÃO INTESTINAL EM ADULTO, RELATO DE CASO E REVISÃO DA LITERATURA
}

\author{
Adult intestinal malrotation, case report and literature review
}

\author{
Ubirajara Rutilio Mendes e Ferreira de ARAÚJO, Imad Izat EL TAWIL
}

\begin{abstract}
Araújo URMF, El Tawil II. Má rotação intestinal em adulto, relato de caso e revisão da literatura. ABCD Arq Bras Cir Dig 2009;22(4):240-2
RESUMO - Introdução - Má rotação intestinal é entidade clínico-cirúrgica que faz parte do cotidiano do cirurgião pediátrico, mas que se torna um desafio diagnóstico quando desenvolve sintomas em adolescentes e adultos. Relato do caso-Mulher deu entrada no hospital com quadro de intensa dor abdominal com três dias de evolução e piora progressiva nas últimas 24 horas. A dor apresentava piora importante após as refeições, quando era acompanhada de náuseas e vômitos. Ao exame físico apresentava-se em bom estado gera, abdômen plano, flácido, ruídos presentes, levemente doloroso à palpação de epigástrio, mas sem sinais de irritação peritoneal. Exames laboratoriais encontravam-se dentro dos limites da normalidade, bem como estudo ultrassonográfico. Não houve melhora clínica apesar do tratamento instituído e optou-se por investigação cirúrgica por tomografia sugerir má rotação intestinal. No intra-operatório observou-se todo o intestino delgado disposto para o lado direito do abdômen e o cólon para o lado esquerdo. Além disto, o jejuno proximal encontrava-se isquêmico e fazendo um volvo de $720^{\circ}$ sobre o eixo dos vasos mesentéricos superiores. Para a correção da anomalia fez-se enterotomia do jejuno proximal, a cerca de $10 \mathrm{~cm}$ do ligamento de Treitz, e desconfecção do volvo, o que cursou com melhora progressiva da isquemia intestinal, permitindo que se fizesse enteroanastomose. Realizou-se ligadura do pedículo da artéria cólica média em sua origem e colectomia direita seguida de anastomose íleo-transversa látero-lateral. A paciente evolui bem. Conclusão - A má rotação intestinal em adultos é doença de difícil diagnóstico primário, devido a não constar entre as hipóteses diagnósticas iniciais do cirurgião geral.

DESCRITORES - Má rotação intestinal. Adultos.
\end{abstract}

\section{INTRODUÇÃO}

A má rotação intestinal é anomalia congênita causada por uma rotação incompleta ou não rotação do intestino no eixo da artéria mesentérica superior durante o desenvolvimento embriológico ${ }^{2}$. Apresenta-se tipicamente nos primeiros meses de vida, mas às vezes pode se surgir tardiamente causando dificuldade e erro no diagnóstico?. Sabe-se que $64 \%$ dos casos tornam-se evidentes clinicamente nos primeiros meses de vida e $82 \%$ até o primeiro ano de vida ${ }^{9}$ porém, alguns autores afirmam que até $90 \%$ dos casos são diagnosticados ao se completar um $\mathrm{ano}^{4}$. A real incidência desta malformação não é conhecida, com estimativas variando de 1:200 a 1:6000 nascidos vivos ${ }^{4}$. Em adultos, ela é de $0,2 \%$, sendo que cerca de $15 \%$ de todos os pacientes com diagnóstico firmado de má rotação intestinal permanecem assintomáticos por toda vida ${ }^{6}$. Por estes motivos, o diagnóstico desta doença faz parte do diaa-dia do cirurgião pediátrico, mas esta hipótese diagnóstica frequentemente fica esquecida nos casos de dor abdominal em adultos ${ }^{1}$.

Trabalho realizado no Ônix Centro Hospitalar, Curitiba, PR, Brasil

Endereço para correspondência: Ubirajara Araújo, e-mail: biraraujo@bol.com.br

\section{RELATO DO CASO}

P.S.Z., 21 anos, sexo feminino, telefonista. Deu entrada no hospital com quadro de intensa dor abdominal com três dias de evolução e piora progressiva nas últimas 24 horas. Referia ser a dor abdominal do tipo difusa, em cólica, porém mais intensa em região epigástrica e com irradiação para dorso. A dor apresentava piora importante após as refeições, quando era acompanhada de náuseas e vômitos. Negava quaisquer outras queixas associadas. Não apresentava nenhuma co-morbidade, mas referia na história mórbida pregressa episódio semelhante há três anos, porém de caráter fugaz e com melhora espontânea com repouso intestinal e medicação anti-espasmódica.

Ao exame físico apresentava-se em bom estado geral, levemente desidratada, normocorada, afebril, com dados vitais estáveis, abdômen plano, flácido, ruídos presentes, levemente doloroso à palpação de epigástrio mas sem sinais de irritação peritoneal.

Foram solicitados exames laboratoriais (hemograma, eletrólitos, avaliação da função hepática e pancreática) que se encontravam dentro dos limites da normalidade, bem como ultrassonografia do abdômen que evidenciou somente distensão de alças intestinais. Iniciaram-se medicações analgésicas e bloqueador de bomba de prótons e solicitouse endoscopia digestiva alta, a qual demonstrou apenas pequena hérnia hiatal. No segundo dia de internamento o quadro clínico permanecia inalterado, mas com maior 
intolerância alimentar; então, foi solicitada tomografia de abdômen. O laudo concluiu tratar-se de má rotação intestinal ao longo do eixo da artéria mesentérica superior, com sinais de sub-oclusão do trato gastrintestinal alto.

Optou-se por conduta cirúrgica através da via laparotômica. No intra-operatório observou-se todo o intestino delgado disposto para o lado direito do abdômen e o cólon para o lado esquerdo. Além disto, o jejuno proximal encontravase isquêmico e fazendo um volvo de $720^{\circ}$ sobre o eixo dos vasos mesentéricos superiores; já o cólon direito estava disposto por trás destes vasos, mas com a sua arcada vascular disposta acima deles. Para a correção da anomalia fez-se enterotomia do jejuno proximal, a cerca de $10 \mathrm{~cm}$ do ângulo duodenojejunal, e desconfecção do volvo, o que cursou com melhora progressiva da isquemia intestinal, permitindo que se fizesse uma enteroanastomose. O cólon direito através da arcada vascular da cólica direita, contudo, comprimia os vasos mesentéricos superiores, fazendo ingurgitamento dos mesmos. Então, realizou-se ligadura do pedículo da artéria cólica média em sua origem e colectomia direita seguida de anastomose íleo-transversa látero-lateral.

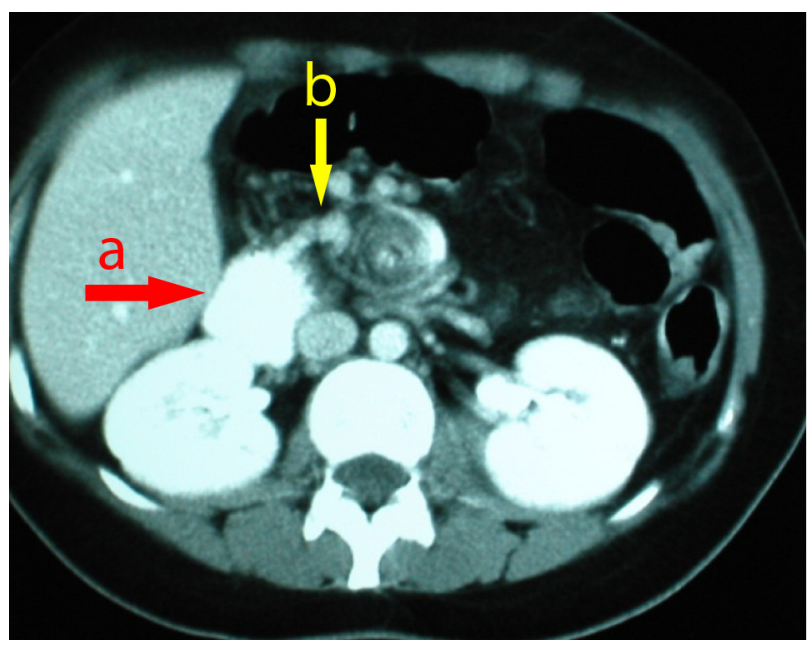

FIGURA 1 - Tomografia computadorizada de abdômen mostrando em a) dilatação e espessamento da parede duodenal e em b) transição duodeno-jejunal deslocada à direita da linha média

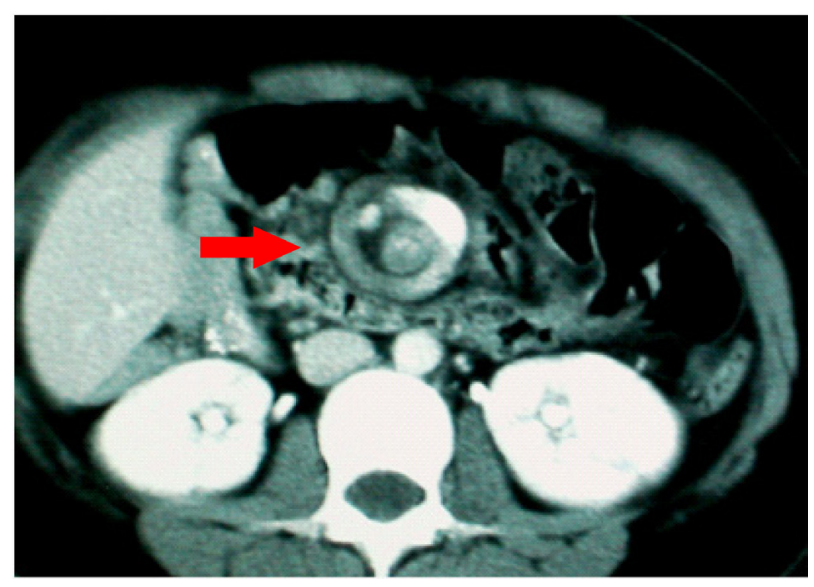

FIGURA 2 - Rotação intestinal de $720^{\circ}$ sobre o eixo dos vasos mesentéricos superiores.
A paciente evolui satisfatoriamente bem, tendo alta hospitalar no $5^{\circ}$ dia de pós-operatório e manteve-se assintomática até o presente momento ( 2 anos de pós-operatório).

\section{DISCUSSÃO}

Segundo alguns autores a grande maioria dos casos de má rotação intestinal em adultos é assintomática ${ }^{4,5,9}$; dentre aqueles que desenvolverão algum sintoma, a evolução pode ocorrer de forma aguda ou crônica. A forma aguda cursa com quadro de vômitos e dor abdominal sem distensão (por se tratar de obstrução alta) que evolui para isquemia e necrose intestinal com peritonite ${ }^{1}$. Isto pode ser causado por obstrução intestinal por volvo ou hérnia interna, com ou sem isquemia intestinal $^{2,5}$. Em geral, com uma boa anamnese descobre-se história prévia de sintomas intermitentes, mas leves ${ }^{1}$. No presente caso, a evolução ocorreu de forma aguda, tendo sido causada por volvo do jejuno com consequente obstrução intestinal. Já nos casos de evolução crônica, os sintomas são inespecíficos, como dor abdominal tipo cólica de caráter intermitente e vômitos recorrentes ${ }^{1,6}$. A causa dessa anomalia é a incorreta fixação do intestino delgado, causando um estreitamento no pedículo dos vasos mesentéricos superiores, o que predispõe o intestino ao volvo ${ }^{5,6}$. Além disto, formamse feixes de peritônio para tentar fixar compensatoriamente o ceco (que está localizado no quadrante superior direito) na parede abdominal; estes feixes cruzam o duodeno mal posicionado e podem causar obstrução ou criar espaços para formação de hérnias internas ${ }^{5,6}$. Estes sintomas vagos tornam o diagnóstico obscuro, retardando-o, e não raro o paciente é atendido por vários médicos, que atribuem as causas das queixas à problemas da motilidade intestinal ou à desordens de origem psiquiátrica antes de esclarecer o correto e definitivo diagnóstico ${ }^{2,3,4,5,6}$.

Quanto ao relatado, o diagnóstico foi elucidado através de tomografia contrastada de abdômen. Este exame vem sendo cada vez mais solicitado e demonstra alterações de parâmetros anatômicos característicos, tais como relação inversa entre os vasos mesentéricos superiores (veia está situada à esquerda da artéria) $)^{1,4,10}$, ausência ou hipoplasia do processo uncinado do pâncreas ${ }^{4,10}$ e alterações na disposição das alças intestinais ${ }^{4}$. Porém, o diagnóstico poderia ter sido feito através de raio-X contrastado do trato gastrintestinal alto, o qual segundo muitos autores, é o exame padrão-ouro para o diagnóstico de má rotação intestinal ${ }^{1,4}$, tendo acurácia superior a $80 \%$ e em alguns estudos atingindo $100 \%{ }^{5}$. O achado mais importante a ser observado é a junção duodeno-jejunal, a qual não cruza a linha média e fica localizada à direita da coluna vertebral ${ }^{1,4,6}$. Além destes, há outros exames para o diagnóstico tais como ultrassonografia de abdômen (também avaliando a relação inversa entre os vasos mesentéricos) $)^{3,6,9,10}$, angiografia $^{3,6}$ e a videolaparoscopia ${ }^{7}$. Nos casos agudos, no entanto, o diagnóstico na maioria das vezes continua a ser feito através da laparotomia ${ }^{3}$.

Finalmente, no que diz respeito ao tratamento, não existe padronização para adultos, sendo que vai depender dos achados intra-operatórios e do tipo de malformação associada ${ }^{4}$. Porém, em geral deve-se fazer uma série de 
manobras chamadas de procedimento de Ladd, que consiste na liberação das aderências existentes, mobilização do duodeno e do cólon direito, liberação e alargamento do pedículo dos vasos mesentéricos superiores e realização de apendicectomia profilática ${ }^{1,2,4,5,8}$. Não há consenso quanto à prevenção de complicações peri e pós-operatórias através da cecopexia ${ }^{4}$. Atualmente, tem-se sugerido a realização do procedimento de Ladd por videolaparoscopia, por ter bons resultados e mínima morbidade ${ }^{4,7,8}$. Segundo alguns estudos, a videolaparoscopia quando comparada ao procedimento convencional, tem significativamente menor tempo de internamento pós-operatório e menor necessidade de analgesia pós-operatória ${ }^{5}$. Com relação aos casos assintomáticos, não há consenso quanto à conduta a ser tomada ${ }^{4}$, há autores que afirmam que não se deve operar o paciente ${ }^{1}$, mas há outros, em contra-partida, que alegam que o tratamento cirúrgico deve ser instituído para prevenir complicações causadas por volvo ou hérnia interna ${ }^{5,6}$.

\section{CONCLUSÃO}

A má rotação intestinal em adultos é doença de difícil diagnóstico primário devido a não constar entre as hipóteses diagnósticas iniciais do cirurgião geral. A sua lembrança é cabível nos quadros arrastados de dor abdominal sem explicação plausível.

Araújo URMF, El Tawil II. Adult intestinal malrotation, case report and literature review. ABCD Arq Bras Cir Dig 2009;22(4):240-2

ABSTRACT - Introduction - Intestinal malrotation is a clinical surgical entity that is present in the everyday practice of the pediatric surgeon. However, it becomes a diagnostic challenge when symptoms develop in adolescents and adults. Case report - A woman presented to the hospital with intense abdominal pain of three days' duration and progressive worsening over the preceding 24 hours. The pain increased markedly after meals, accompanied by nausea and vomiting. On physical examination, the patient was in good general health, her abdomen was flat, flaccid, with normal bowel sounds, and tender to palpation of the epigastrium, yet with no signs of peritoneal irritation. Laboratory test results were within the limits of normal, as was ultrasonography. No clinical improvement was achieved despite the treatment instituted; surgical exploration was chosen as tomography was suggestive of intestinal malrotation. Intraoperatively, all the small intestine was found to be positioned to the right side of the abdomen and the colon, to the left side. In addition, the proximal jejunum was ischemic and forming a volvulus of $720^{\circ}$ over the axis of the superior mesenteric vessels. In order to correct the anomaly, enterotomy of the proximal jejunum was performed at approximately $10 \mathrm{~cm}$ from the ligament of Treitz, and the volvulus was corrected. This promoted a progressive improvement of the intestinal ischemia, which made enteroanastomosis possible. The middle colic artery pedicle was ligated at its root and a right colectomy was performed, followed by a side-to-side ileo-transverse anastomosis. The patient is doing well. Conclusion - Intestinal malrotation in adults is a condition of difficult primary diagnosis, since it is not among the initial diagnostic hypotheses of the general surgeon.

HEADINGS - Intestinal malrotation. Adults.

\section{REFERÊNCIAS}

1. Dietz DW, Walsh RM, Grudfest-Broniatowski S, Lavery IC, et al. Intestinal malrotation: a rare but important cause of bowel obstruction in adults. Dis Colon Rectum 2002, 45(10):1381-6.

2. Gamblin TC, Stephens Jr RE, Jhonson RK, Rothwell M. Adult malrotation: a case report and review of the literature. Curr Surg 2003, 60(5):517-20.

3. Jayathillake A, Shields MA. Malrotation with volvus: a rare cause of acute bowel obstruction in na adult. ANZ J Surg 2005, 75(9):831-3.

4. Krapfer SA, Rappold JF. Intestinal malrotation - not just the pedriatric surgeon's problem. J Am Coll Surg 2004, 199(4):628-35.

5. Matzke GM, Dozois EJ, Larson DW, Moir CR. Surgical management of intestinal malrotation in adults: comparative results for open and laparoscopic Ladd procedures. Surg Endosc 2005, 19(10):1416-9.
6. Maxson RT, Franklin PA, Wagner CW. Malrotation in the older child; surgical management, treatment, and outcome. Am Surg 1995, 61(2):135-8.

7. Mazziotti MV, Strasberg SM, Langer JC. Intestinal rotation abnormalities without volvulus: the role of laparoscopy. J Am Coll Surg 1997, 185(2):172-6.

8. Seymour NE, Andersen DK . Laparoscopic treatment of intestinal malrotation in adults. JSLS 2005, 9(3):298-301

9. Vukie Z. Presentation of intestinal malrotation syndromes in older children and adults: report of three cases. Croat Med J 1998, 39(4):455-7.

10. Zissin R, Rathaus V, Oscadchy A, Kots E, et al. Intestinal malrotation as na incidental finding on CT in adults. Abdom Imaging 1999, 24(6):550-5.

Fonte de financiamento: não há Conflito de interesse: não há Recebido para publicação:07/09/2008 Aceito para publicação: 14/10/2008 